\title{
Math-Net.Ru
}

А. В. Чашкин, О сложности и глубине схем, реализующих частичные булевы функции, Дискрет. матем., 1997, том 9, выпуск 2, 53-58

DOI: https://doi.org/10.4213/dm463

Использование Общероссийского математического портала Math-Net.Ru подразумевает, что вы прочитали и согласны с пользовательским соглашением http://www.mathnet.ru/rus/agreement

Параметры загрузки:

IP : 54.164 .48 .24

26 апреля 2023 г., 12:15:01

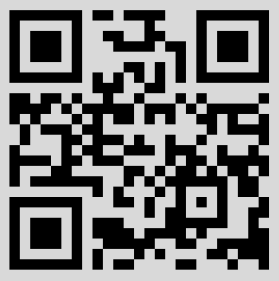




\title{
Дискретная математика
}

том 9 выпуск $2 * 1997$

УДК 519.7

\section{О сложности и глубине схем, реализующих частичные булевы функщии}

\author{
(c) 1997 г. А. В. Чашкин
}

\begin{abstract}
Изучается сложность и глубина схем, реализующих частичные булевы функции в предположении $F P \neq N C$. При этом предположении устанавливается существование частичных булевых функщий, для которых не существует схем, сложность и глубина которых одновременно близки к минимально возможным, т.е. у любой схемы, реализующей одну из таких функций, либо глубина, либо сложность значительно превосходит соответственно либо глубину, либо сложность реализуемой функции.
\end{abstract}

Сложностью $L(f)$ булевой функции $f$ назовем сложность $L(S)$ минимальной схемы $S$, реализующей эту функцию в базисе $\{\&, \vee, \neg\}$. Глубиной $d(S)$ схемы $S$ назовем максимальное число элементов схемы $S$, лежащих на какой-либо цепи, соединяющей один из входных полюсов $S$ с ее выходным полюсом. Глубиной $d(f)$ функции $f$ назовем глубину самой “неглубокой” схемы, реализующей эту функцию, т.е. $d(f)=\min d(S)$, где минимум берется по всем схемам, реализующим $f$.

В работе изучается сложность и глубина схем, реализующих частичные булевы функции в предположении $F P \neq N C$. Пусть $n$ - число переменных рассматриваемых функций. Тогда $F P$ - класс функций, вычисляемых последовательно за полиномиальное относительно $n$ время, и $N C$ - класс функций, вычисляемых параллельно за время $\left(\log _{2} n\right)^{O(1)}$ с полиномиальной, также относительно $n$, сложностью. В терминах булевых функций и схем из функциональных элементов, реализующих эти функции, гипотеза $F P \neq N C$ может быть сформулирована в виде следующего предположения $A$ :

для любых постоянных $c_{1}, c_{2}, c_{3}$ найдется такая постоянная $n\left(c_{1}, c_{2}, c_{3}\right)>0$, что при любом $n \geqslant n\left(c_{1}, c_{2}, c_{3}\right)$ существует такая функция $f:\{0,1\}^{n} \rightarrow\{0,1\}$, что

$-L(f) \leqslant n^{c_{1}}$

- если схема $S$ реализует функцию $f$, и $L(S) \leqslant(L(f))^{c_{2}}$, то $d(S) \geqslant\left(\log _{n}\right)^{c_{3}}$.

Из доказываемой в работе теоремы следует существование частичных функций, для которых не существует схем, сложность и глубина которых одновременно близки к минимально возможным, т.е. у любой схемы, реализующей одну из таких функций, либо глубина, либо сложность значительно превосходит соответственно либо глубину, либо сложность реализуемой функции.

Введем несколько необходимых в дальнейшем определений. Пусть $D_{1} \subset D_{2} \subseteq$ $\{0,1\}^{n}$. Функцию $g: D_{1} \rightarrow\{0,1\}$ назовем сужением функции $h: D_{2} \rightarrow\{0,1\}$ на 
область $D_{1}$, если $g(x)=h(x)$ при любом $x \in D_{1}$. Сужение функции $f$ на область $D$ обозначим $f_{D}$, т.е. для функций $g$ и $h$ справедливо равенство $g=h_{D_{1}}$. Пусть, далее, $N(L, n)$ - число неизоморфных схем в базисе $\{\&, \vee, \neg\}$, имеющих $n$ входов, один выход, и сложность, не превосходящую $L$.

Основным результатом работы является следующая теорема.

Теорема. Пусть справедливо предположение А. Тогда для любых постоянных $c_{1} u$ $c_{2}$ найдется такая постоянная $n\left(c_{1}, c_{2}\right)$, что при любом $n \geqslant n\left(c_{1}, c_{2}\right)$ существует такая область $D \subseteq\{0,1\}^{n}$ и такая функция $f: D \rightarrow\{0,1\}$, что, если схема $S$ реализует функцию $f$ u $L(S) \leqslant(L(f))^{c_{1}}, \bmod d(S) \geqslant(d(f))^{c_{2}}$.

Доказательство теоремы основано на леммах 2 и 3 . Доказательство леммы 2 легко извлекается из доказательства теоремы 1 из [1]. В доказательстве леммы 3 используется лемма 1, доказанная в [2] (см. лемму 6 в [2]).

Лемма 1. Пусть $a$, с и $d-$ положительные челые числа, и $b-$ положительное число. Пусть $\left\lfloor(2 a b)^{1 / 2}\right\rfloor \leqslant d \leqslant a / 2 u a>2 b>5$. Тогда максимальное $c$, удовлетворятоее неравенству

$$
\left(\begin{array}{l}
a \\
d
\end{array}\right) /\left(\begin{array}{c}
a-c \\
d
\end{array}\right) \leqslant \exp b
$$

не превосходит $d$.

Лемма 2. Пусть $D \subseteq\{0,1\}^{n},|D| \geqslant n, f: D \rightarrow\{0,1\}$. Тогда существует такая постоянная $c_{4}$, что

$$
d(f) \leqslant c_{4} \log _{2}|D|
$$

Лемма 3. Пусть $D \subseteq\{0,1\}^{n}, f: D \rightarrow\{0,1\}, L \geqslant L(f) u$

$$
D \geqslant 2 \log _{2}(N((L-4) / 3, n)) \text {. }
$$

Пусть для глубины любой схемы $S$, реализующей $f$ со сложностъю $L(S) \leqslant L$, справедливо неравенство $d(S) \geqslant k$. Тогда существует такая область $D^{\prime} \subseteq D$, что

$$
\left|D^{\prime}\right| \leqslant 2\left(2|D| \log _{2}(N((L-4) / 3, n))\right)^{1 / 2},
$$

$u$ глубина любой схемь $S$, реализующей функцию $f_{D^{\prime}}$ со сложностью $L(S) \leqslant(L-4) / 3$, удовлетворяет неравенству

$$
d(S) \geqslant k-3
$$

Доказателъство. Введем целый положительный параметр $d$. Положим

$$
d=\left\lfloor\left(2|D| \log _{2}(N((L-4) / 3, n))^{1 / 2}\right\rfloor .\right.
$$

Рассмотрим множество областей

$$
M_{d}=\left\{V_{i}\left|V_{i} \subseteq D,\right| V_{i} \mid=d\right\},
$$

и сужения функции $f$ на области этого множества. Возможны два случая: 
- среди областей $V_{i} \in M_{d}$ существует такая область $V_{j}$, что глубина любой схемы $S$, реализующей функцию $f_{V_{j}}$ со сложностью $L(S) \leqslant(L-4) / 3$, удовлетворяет неравенству $d(S) \geqslant k-3$;

- для любой области $V_{i}$ найдется схема $S$, реализующая функцию $f_{V_{i}}$ со сложностью $L(S) \leqslant(L-4) / 3$ и глубиной $d(S) \leqslant k-4$.

В первом случае утверждение леммы очевидно. В качестве $D^{\prime}$ достаточно взять область $V_{j}$.

Рассмотрим второй случай. Так как

$$
\left|M_{d}\right|=\left(\begin{array}{c}
|D| \\
d
\end{array}\right)
$$

в этом случае существует схема $S_{1}$, сложность и глубина которой не превосходят, соответственно, $(L-4) / 3$ и $k-4$, реализующая на $D$ некоторую булеву функцию $h$, совпадающую с $f$ не менее чем на $\left(\begin{array}{c}|D| \\ d\end{array}\right) / N((L-4) / 3, n)$ областях множества $M_{d}$. Пусть

$$
V=D \backslash\left(\bigcup_{i} V_{i}\right),
$$

где объединение берется по всем областям $V_{i}$, на которых $f=h$. Оценим сверху мощность $R$ множества $V$. Очевидно, $R$ удовлетворяет неравенству

$$
\left(\begin{array}{c}
|D| \\
d
\end{array}\right) /\left(\begin{array}{c}
|D|-R \\
d
\end{array}\right) \leqslant 2^{\log _{2} N((L-4) / 3, n)} .
$$

Из леммы 1 и неравенства (1) следует, что

$$
R \leqslant d
$$

Введем новый параметр $d^{\prime}$. Положим

$$
d^{\prime}=\left\lfloor\left(2(|D|-R) \log _{2}(N((L-4) / 3, n))^{1 / 2}\right\rfloor .\right.
$$

Рассмотрим новое множество областей

$$
M_{d^{\prime}}=\left\{V_{i}^{\prime}\left|V_{i}^{\prime} \subseteq D, V \subset V_{i}^{\prime},\right| V_{i}^{\prime} \mid=d^{\prime}+R\right\}
$$

Как и ранее, возможны два случая:

- среди областей $V^{\prime} \in M_{d^{\prime}}$ существует такая область $V_{j}^{\prime}$, что глубина любой схемы $S$, реализующей функцию $f_{V_{j}^{\prime}}$ со сложностью $L(S) \leqslant(L-4) / 3$, удовлетворяет неравенству $d(S) \geqslant k-3$;

- для любой области $V_{i}^{\prime}$ найдется схема $S$, реализующая функцию $f_{V_{i}^{\prime}}$ со сложностью $L(S) \leqslant(L-4) / 3$ и глубиной $d(S) \leqslant k-4$.

В первом случае в качестве $D^{\prime}$ можно взять область $V_{j}^{\prime}$, так как $\left|V_{j}^{\prime}\right| \leqslant 2 d$ в силу (2). Если имеет место второй случай, то вновь воспользуемся леммой 1 . Нетрудно показать, что в этом случае существует некоторая булева функция $g$, определенная на области $D$ и совпадающая с функцией $f$ не менее чем на $|D|-R$ наборах 
области $D$, причем существует схема $S_{2}$, реализующая функцию $g$ со сложностью, не превосходящей $(L-4) / 3$, и имеющая глубину, не превосходящую $k-4$. Пусть наборы, на которых совпадают значения функций $f$ и $g$, составляют область $W$, и пусть

$$
V^{\prime}=D \backslash W .
$$

Рассмотрим далее такую функцию $z: D \rightarrow\{0,1\}$, значения которой при любом $x \in$ $V \cup V^{\prime}$ совпадают с соответствующими значениями функции $f$. Нетрудно убедиться в справедливости равенства

$$
f=h y \vee h z \vee g z=h(g \vee z) \vee y z .
$$

Предположим, что существует схема $S_{3}$, реализующая $z$ со сложностью, не превосходящей $(L-4) / 3$, и имеющая глубину, не превосходящую $k-4$. В этом случае схема $S$, состоящая из схем $S_{1}, S_{2}, S_{3}$, выходы которых присоединены ко входам схемы, реализуюшей функцию голосования, реализует функцию $f$. Нетрудно убедиться в том, что

$$
L(S) \leqslant L, \quad d(S) \leqslant k-1 .
$$

Приходим к противоречию. Так как $\left|V \cup V^{\prime}\right| \leqslant 2 d$, лемма 3 доказана.

Доказательство теоремы. Пусть $c_{1}, c_{2}$ - произвольные постоянные, большие единицы. В соответствии с предположением $A$, существует такая постоянная $n\left(c_{1}, c_{2}\right)$, что при любом $n \geqslant n\left(c_{1}, c_{2}\right)$ существует функция $f:\{0,1\}^{n} \rightarrow\{0,1\}$, сложность которой не превосходит $n^{2}$, а глубина любой схемы $S$, реализующей $f$ со сложностью $L(S) \leqslant n^{2 c_{1}+2}$, ограничена снизу величиной $\left(\left(\log _{2} n\right)^{c_{2}+1}+3 \log _{2} n\right)$. Полагая

$$
L=n^{2 c_{1}+2}, \quad k=\left(\left(\log _{2} n\right)^{c_{2}+1}+3 \log _{2} n\right),
$$

и применяя лемму 3 к $f$, получаем область $V_{1} \subseteq\{0,1\}^{n}$ и сужение $f_{V_{1}}$ функции $f$ на эту область, причем глубина любой схемы $S$, реализующей сужение $f_{V_{1}}$, ограничена снизу величиной $k-3$, если $L(S) \leqslant(L-4) / 3$. К полученному сужению вновь применим лемму 3 , и т.д. После $s$-го применения леммы 3 (предположим, что это возможно) получим последовательность вложенных областей

$$
V_{s} \subseteq V_{s-1} \subseteq \cdots \subseteq V_{1} \subseteq V_{0}=\{0,1\}^{n},
$$

последовательность

$$
f_{V_{s}}, f_{V_{s-1}}, \ldots, f_{V_{1}}, f_{V_{0}}=f
$$

сужений функции $f$ на эти области, и последовательность

$$
L_{s}, L_{s-1}, \ldots L_{1}, L_{0}=L
$$

такие, что

$$
\begin{gathered}
\left|V_{i}\right| \leqslant 2\left(2\left|V_{i-1}\right| \log _{2} N\left(\left(L_{i-1}-4\right) / 3, n\right)\right)^{1 / 2} \\
L_{i} \geqslant\left(L_{I-1}-4\right) / 3
\end{gathered}
$$

и глубина любой схемы $S$, реализующей $f_{V_{i}}$, ограничена снизу величиной

$$
k-3 i,
$$


если $L(S)<L_{i}$.

Оценим $L_{t}$ для минимального $t$, при котором

$$
\left.\left|V_{t}\right| \leqslant 16 \log _{2} N\left(L_{0}, n\right)\right) .
$$

Так как $L_{i} \leqslant L_{0}$, заменяя (3) более слабым неравенством

$$
\left|V_{i}\right| \leqslant 2\left(2\left|V_{i-1}\right| \log _{2} N\left(\left(L_{0}\right)-4\right) / 3, n\right)^{1 / 2},
$$

видим, что

$$
16 \log _{2} N\left(L_{0}, n\right)<\left|V_{t-1}\right| \leqslant 4\left(2^{n}\right)^{1 / 2^{t-1}} \log _{2} N\left(L_{0}, n\right) ;
$$

следовательно,

$$
2^{t}<n
$$

Из (4) и (6) следует, что

$$
L_{t} \geqslant n^{-\log _{2} 3} L_{0}-6>n^{2 c_{1}} .
$$

В силу леммы 2 и леммы Д.3 из [3]

$$
d\left(f_{V_{t}}\right) \leqslant c_{4} \log _{2}\left|V_{t}\right| \leqslant c_{4} \log _{2}\left(16 \log _{2} N\left(L_{0}, n\right)\right) \leqslant c_{4} \log _{2}\left(16 c_{5} L_{0} \log _{2} L_{0}\right) \leqslant c_{6} \log _{2} n,
$$

где $c_{5}$ и $c_{6}$ - некоторые постоянные.

Из (5) и (7) следует, что глубина любой схемы $S$, реализующей функцию $f_{V_{t}}$, ограничена снизу величиной $\left(\log _{2} n\right)^{c_{2}}$, если $L(S)<L_{t}$. Очевидно, что, начиная с некоторого $n_{0}$, имеет место неравенство

$$
\left(\log _{2} n\right)^{c_{2}+1} \geqslant\left(c_{6} \log _{2} n\right)^{c_{2}} .
$$

Следовательно,

$$
\left(d\left(f_{V_{t}}\right)\right)^{c_{2}} \leqslant\left(\log _{2} n\right)^{c_{2}+1} .
$$

Поэтому глубина любой схемы $S$, реализующей функцию $f_{V_{t}}$, ограничена снизу величиной $\left(d\left(f_{V_{t}}\right)\right)^{c_{2}}$, если $L(S)<L_{t}$.

Таким образом, теорема доказана для $s \geqslant t-1$. Для завершения доказательства покажем, что лемма 3 может быть использована не менее $t-1$ раза. Предположим, что это не так, т.е. $s<t-1$. В этом случае лемма 3 неприменима к функции $f_{V_{s}}$. Так как

$$
L_{s} \geqslant L_{t} \geqslant n^{2 c_{1}}>n,
$$

лемма 3 не может быть применена к функции $f_{V s}$ вследствие невыполнения условия

$$
\left|V_{s}\right| \geqslant 2 \log _{2} N\left(\left(L_{s-1}-4\right) / 3, n\right) \text {. }
$$

Однако цепочка неравенств

$$
16 \log _{2} N\left(L_{0}, n\right)<\left|V_{t-1}\right| \leqslant\left|V_{s}\right|<2 \log _{2} N\left(\left(L_{s-1}-4\right) / 3, n\right) \leqslant 2 \log _{2} N\left(L_{0}, n\right) .
$$

приводит к противоречию. Следовательно, $s \geqslant t-1$. Теорема доказана. 


\section{Список литературы}

1. Андреев А. Е. О сложности реализации частичных булевых функций схемами из функциональных элементов. Дискретная математика (1989) 1, №4, 36-45.

2. Чашкин А. В. О сложности сужений булевых функций. Дискретная математика (1996) 8, №2, 133-150.

3. Лупанов О. Б. Об одном подходе к синтезу управляющих систем - принципе локального кодирования. Пробл. киберн. (1965) 14, 31-110.

Статья поступила 30.09.1994. 